\title{
Pre-implantation genetic screening and diagnosis: what doctors should know
}

\author{
Rastreio ou diagnóstico genético pré-implantação: \\ o que os médicos devem saber
}

Editorial

\section{Introduction}

Trends toward delayed childbearing have resulted in an increasing number of women of advanced maternal age (AMA) seeking to become pregnant and in a consequent increase in demand for assisted reproductive technology, most commonly in-vitro fertilization (IVF) ${ }^{1}$. In such women, the proportion of aneuploid embryos can exceed $60 \%$, with a risk of miscarriage of about $40 \%$, potentially resulting in significant emotional and financial hardship for affected couples. In the last 30 years, genetic testing techniques have been developed to identify chromosomally normal embryos in vitro, thereby potentially increasing the proportion of successful cycles with elective single-embryo transfer, and minimizing twin-pregnancy complications and miscarriages ${ }^{2}$. This testing is termed "pre-implantation genetic screening"(PGS), in contrast to pre-implantation genetic diagnosis, in which testing is performed for specific genetic defects. Collectively, these techniques are called pre-implantation genetic testing.

Today, PGS technologies have evolved to include screening of all 24 chromosomes (22 pairs of autosomes and the 2 sex chromosomes). Ongoing pregnancy rates of about $60 \%$ following single embryo transfer have been described in couples with a maternal age of 38 years whose embryos have undergone PGS. It has not, however, been definitively established that the cumulative delivery rates are better with PGS, although it has been argued that the reduction in miscarriage rates and maternal and neonatal complications due to multiple pregnancies justifies the expense of this technology ${ }^{2}$.

How can clinicians assist in ensuring couples make informed decisions? Some points in answer to this question are discussed below.

To whom should be offered pre-implantation genetic testing?

The primary indications for pre-implantation testing are as follows:

\section{Pre-implantation genetic screening}

Commonly quoted indications for PGS include advanced maternal age, repeated implantation failure, recurrent miscarriage, severe male factor infertility, or subfertility

Correspondence

Fabricio da Silva Costo Royal Women's Hospital 20 Flemington Road, Parkville VIC 305 Melbourne, Victoria, Australio

Received $04 / 15 / 2014$
Department of Perinatal Medicine, The Royal Women's Hospital - Melbourne, Victoria, Australia. 'Department of Perinatal Medicine, Royal Women's Hospital - Melbourne, Victoria, Australia.

2Department of Obstetrics and Gynaecology, University of Melbourne - Melbourne, Victoria, Australia. 32Department of Public Health, Universidade Estadual do Ceará - Fortaleza (CE), Brazil.

${ }^{4}$ Monash Ultrasound for Women - Melbourne, Victoria, Australia. 
(those who experience unrecognized embryonic losses and who are labelled clinically as infertile) ${ }^{3,4}$. The intention of PGS is to improve live birth rates after IVF treatment by exclude embryos with aneuploidy prior to transfer. The development of advanced technologies allows analysis of all 23 pairs of chromosomes (22 pairs of autosomes and the 2 sex chromosomes) known as " 24 chromosomes" analysis ${ }^{2}$. PGS is a screening method, performed for the couple who has no increased risk of having a child with a specific inherited disease ${ }^{5}$. Although randomised controlled trials have shown some increase in pregnancy rates after the use of PGS, they have not yet demonstrated an increase in live birth rate, so its use has not been completely validated ${ }^{5-7}$.

\section{Pre-implantation genetic diagnosis}

Pre-implantation genetic diagnosis (PGD) allows couples with a family history of monogenic disorders, $x$-linked diseases and known chromosomal abnormality to avoid the transfer of embryoswith these specific genetic disorders ${ }^{3}$. PGD technology have three primary applications:

- Single gene disorders - Autosomal dominant diseases, such as myotonic dystrophy type I (MD1), neurofibromatosis and Charcot-Marie-Tooth disease; autosomal recessive disorders, as beta-thalassaemia/sickle cell disorders (with or without HLA typing), cystic fibrosis and spinal muscular atrophy (SMA); and X-linked recessive diseases (in which PGD has been conducted for a specific diagnosis),including Fragile X syndrome, Duchenne/Becker muscular dystrophy, and haemophilia $\mathrm{A}$ and $\mathrm{B}^{5}$.

- Chromosome rearrangements - Constitutional chromosomal abnormalities are present in up to $0.9 \%$ in newborns, and are associated with 50-60\% of first trimester miscarriages. Most of these aneuploidies are a result of a meiotic non-disjunction event, but about 1/500 individuals carry a balanced structural rearrangement as reciprocal and Robertsonian translocations. Although most present with normal phenotypes, they often suffer from repeated spontaneous abortions and/or fertility problems, and have an increased risk of delivering children with congenital anomalies and/or intellectual disability. Several studies have shown that PGD improves the pregnancy outcome for translocation carriers, especially in patients with recurrent pregnancy losses ${ }^{4,8,9}$.

- PGD-HLA - People affected by malignant conditions, such as leukaemia, lymphoma or some other disorders as beta-thalassaemia, sickle cell anaemia, Fanconi anaemia, Wiscott-Aldrich syndrome, X-linked adrenoleukodystrophy and hypoimmunoglobulin syndromes, may benefit from allogenic haematopoietic stem cell transplantation (allo-HSCT), using an HLA-matched, related donor, most often a sibling child. PGD techniques are helpful in two situations: (a) one child has a non-inherited disease such as leukaemia and the parents want to have PGD with HLA typing alone to allow the newborn to be a donor to the sick child; or (b) one child has a heritable disorder and the parents need PGD in order to avoid another affected child, and, at the same time, HLA typing brings the hope of saving the already affected sibling. For ethical reasons, in some countries, only the latter procedure is deemed acceptable $e^{4,9-11}$.

\section{At what stage of embryonic development can testing be performed?}

There are three approaches for pre-implantation genetic testing used to evaluate the DNA of embryos before day 6 of conception $^{4,5}$. They are the following:

- Polar-body biopsy (assessing female gametes), in which oocyte genotype can be deduced by analysis of the first and/or second polar body. Oocytes considered genetically "normal" can then be utilised for IVF. As it is performed before fertilisation, polar-body analysis offers the possibility of preconception diagnosis without reduction in cell number of the embryo. One clear disadvantage is the inability to assess paternal genotype. However, as $95 \%$ of aneuploidies arise in maternal meiosis, there is little loss in efficiency for aneuploidy testing.

- Blastomere biopsy (aspiration), assessing the 3-day, six- to eight-cell cleavage embryo, in which the zonapellucida is traversed to extract one or two cells.

- Trophectoderm biopsy, assessing the 5- to 6-day, 120-cell blastocyst. Because more cells can be removed at this stage, it potentially facilitates more accurate diagnosis. These additional 2-3 days in culture, beyond that required for an eight-cell embryo, allow some time for natural deselection of non-progressing or aneuploid embryos.

\section{What are the laboratory techniques available for pre-implantation genetic testing?}

Pre-implantation genetic screening was initially performed by fluorescent in-situ hybridisation (FISH) analysis, while pre-implantation genetic diagnosiswas first performed by either FISH or a PCR-based method. Probes are adjusted in order to obtain information about sexing (X-linked disorders), structural chromosomal aberrations, single 
gene disorders, specific translocations or aneuploidy screening (especially for the most commonly affected chromosomes:13, 18, 21, 16, 22, X and $\mathrm{Y}$ ) and single gene disorders 5 .

New technologies are based in personal genomics: comparative genomic hybridization (CGH), microarray-based CGH, multiplex real-time polymerase chain reactions (PCR), digital PCR, real time PCR, single nucleotide polymorphism (SNP) and next generation sequencing $(\mathrm{NGS})^{12}$. Such technologies require a refined laboratory setting, for example the availability of a $5 \%$ oxygen environment, in addition to sizeable resource requirements for incubators, skilled technicians, biologists and embryologists. One of today's challenges is to perform multiple analyses on each embryo, not only for multiple mutations, butfor a combination of diagnosis and aneuploidy screening, perhaps thereby creating a broader testing platform that can be used in all patients 5 .

Most contemporary genetic laboratory technologies can provide results within 24 hours of specimen receipt. Considering that most IVF centers do not have their own genetic laboratories, blastomere biopsy on day 3 provides the diagnostic laboratory sufficient time to analyze and report back the results to facilitate a fresh transfer, but biopsies at this cleavage stage significantly impair human embryonic implantation potential ${ }^{13}$. Some centers have "in-house" laboratories available that may perform genetic analyses in as little as 4 hours, allowing trophectoderm biopsy at the blastocyst stage and a subsequent fresh transfer. A randomized controlled trial has shown that blastocyst biopsy at day 5-6 with comprehensive chromosome screening and fresh embryo transfersignificantly increases in vitro fertilization implantation and delivery rates ${ }^{14}$ while another demonstrated that trophectoderm biopsy with CCS and delayed transfer promises to be a major advance for older women to achieve best maternal and neonatal outcomes ${ }^{2}$. These positive findings await confirmation in further appropriately powered trials.

What are the limitations of pre-implantation genetic testing? What are the safety and ethical concerns of which doctors and couples should be aware?

The highest utilization for pre-implantation techniques at present is aneuploidy screening. Physicians must be aware that the timing of the biopsy is associated with different levels of accuracy in the identification of genetic errors for which the embryos are being screened. Biopsy at the polar body stage provides the earliest specimen, allowing more time for laboratory analysis, and is generally accepted as being less invasive because removal of any portion of the actual embryo is not required. On the other hand, screening too early in development might miss critical errors that could impact on the reproductive potential of the embryo. Conventionally, embryonic aneuploidy has been considered to result almost exclusively from nondisjunction in meiosis I. If this is the case, then nearly all aneuploidy could be identified by assessing the chromosomal complement within the first $\mathrm{PB}^{15,16}$.

Recent studies show that slightly more than $33 \%$ of embryonic aneuploidy may be attributed to errors in meiosis I, and not all are secondary to nondisjunction. Many of the errors are due to premature separation of sister chromatids (PSSC).A recent detailed analysis of this issue found that $90 \%$ of meiosis I errors in the oocyte are PSSC. Nondisjunction still occurs, but may represent only $10 \%$ of the errors. As a result, when reciprocal errors occur for a given chromosomein the first and second PB, $90 \%$ of the embryos will be euploid ${ }^{15,17}$. Thus, with $55 \%$ of PB abnormalities $(10 \%$ from nondisjunction and the remaining $45 \%$ from PSSC), the presence of an abnormal PB screening result provides no information about whether the embryo is euploid or aneuploid. This is why the evaluation of PBs provides suboptimal levels of screening accuracy and the potential for misdiagnoses or non-diagnostic findings is substantial ${ }^{15,16}$.

Biopsying the embryo is more invasive, but overcomes many of the limitations of PB biopsy.As it occurs after thecompletion of meiosis, maternal and paternal meiotic errors should generally be detected. Embryo biopsy may also detect some mitotic errors, while identification of mosaicism depends on what portion a mosaic embryo is impacted by the abnormality. Embryo biopsy may be performed at the cleavage stage (day 3) or at the blastocyst stage (day 5 or 6) of in-vitro development. The decision regarding the day on which to perform the biopsy should be based on the risks and benefits tailored to individual patients' circumstances.

A major limitation to the efficiency of embryonic aneuploidy screening is embryonic mosaicism. Approximately $29 \%$ of all embryos may be mosaic, but many of these will not implant successfully. Of those that do implant, some will subsequently miscarry, but a small percentage may be ongoing, so even women who have undergone pre-implantation aneuploidy screening should still follow routine local protocols for antenatal aneuploidy screening. There are no data supporting whether either day 3 (cleavage estage) or day 5 (trophectoderm) biopsy improves the detection of mosaic embryos. Biopsy at the cleavage stage may reduce implantation rates from 50 to 30\% (relative reduction of 39\%), while biopsy atthe blastocyst stage does not affect the probability thatan embryo will implant and progress to delivery, and therefore appears to be safer ${ }^{15,18}$. 
A meta-analysis with nine RCTs comparing IVF with and without PGS using cleavage-stage biopsy and FISH ('conventional' PGS) showed a significantly lower birth rate for women of advanced maternal age in the PGS group (risk difference: $-0.08 ; 95 \% \mathrm{CI}-0.13--0.03)^{3}$. For a live birth rate of $26 \%$ after IVF without PGS, the rate would be between 13 and 23\% when PGS is used. Trials in which PGS was offered to women with a good prognosis and to women with repeated implantation failures identified similar outcomes. In women of advanced maternal age, conventional PGS significantly lowers the live birth rate, and, as such, the authors concluded that there is no evidence of a beneficial effect of these PGS techniques on the live birth rate after IVF. Mosaicism is thought to be one of the leading causes of the inefficacy of PGS ${ }^{19}$.

Although the trophectoderm biopsy is safer for the embryo, Robertsonian translocations and the majority of reciprocal translocations are identified reliably by FISH, and so it may be better to perform a biopsy at the cleavage stage followed by FISH analysis in these exceptional cases ${ }^{5,15,18}$. The safety of trophectoderm biopsy also needs to be carefully evaluated, because there is a potential risk that placental function and neonatal health could be adversely affected ${ }^{20}$.

Finally, a high proportion of embryo biopsies returns an inconclusive result (approximately one in six). This limits the capacity for PGD to diagnose single gene defects, success of which depends on the nature of the genetic disorder, the linked markers, and assays available $e^{21,22}$.

In order to determine both the negative and positive predictive values for comprehensive chromosome screening (CCS) results for clinical outcome, data were obtained from two prospective, double-blinded, non-selection studies with a total of 255 IVF-derived human embryos cultured and selected for transfer without influence from CCS analysis. Embryos were biopsied before transfer, including 113 blastomeres at the cleavage stage and 142 trophectoderm biopsies at the blastocyst stage. CCS was highly predictive of clinical outcome, with $96 \%$ of aneuploid-predicted embryos failing to sustain implantation and $41 \%$ of embryos predicted to be euploid implanting successfully. These non-selection data provide the first prospective, blinded evidence directly measuring the predictive value of aneuploidy screening for clinical outcome. The conclusion was that clinical error rate of aneuploidy designation is very low (4\%), whereas implantation and delivery rates of euploid embryos are relative increased to the entire cohort of transferred embryos ${ }^{16}$.

A prospective study evaluating 563 live births, 18 still births, and 9 neonatal deaths which had undergone preimplantation genetic testing was compared to another study that analysed ICSI offspring who has not had such testing. About $50 \%$ of the first group were at risk of a single-gene disorder and underwent PGD, whereas the remainder underwent aneuploidy testing. Structural malformations were found in $2.13 \%$ of those undergoing pre-implantation genetic testing and $3.38 \%$ in those conceived following ICSI alone. No differences were observed between offspring resulting from single-gene PGD and aneuploidy testing by PGS ${ }^{23}$. This cohort did not show an increased rate of anomalies overall, or disproportionate clustering of anomalies in any given organ system. Information about the effects of cryopreservation on biopsied embryos is still required, but pre-implantation genetic testing certainly seems safe for use in fresh cycles ${ }^{4}$.

Whole genome scanning technology is accompanied by significant ethical implications. Array CGH allows sexing of embryos, raising concerns about social sex selection, which is illegal in many countries. SNP arrays will identify predispositions for disease that may require detailed counseling. PGD for HLA matching and late-onset disorders has resulted in much ethical debate and is prohibited in many countries. It is also possible to identify features that do not cause disease, but may lead to social advantage (e.g. height); selecting embryos on such grounds will inevitably provoke controversy ${ }^{18,24}$.

Ethical concerns about screening and potential termination of an intrauterine pregnancy after prenatal tests as chorionic villus sampling (CVS), amniocentesis or non-invasive prenatal test (NIPT) are well known ${ }^{21}$. This discussionis likely todiffer from the ethical concerns of pre-implantation genetic testing, which affords parents the opportunity to select embryos for transfer, rather than discontinue an existing pregnancy ${ }^{25}$. However, a common element is the social construct of disability, and the concept that individuals labelled as "disabled" can in fact improve society with their inclusion, rather than being considered burdensome ${ }^{26}$.

Costs of assisted reproductive techniques are high all over the world, and the inclusion of PGS/PGD only increases this cost. In countries where IVF procedures are not publicly funded or covered by insurance, these expenses can be prohibitive for many couples. As a consequence, the clinical recommendation to have such testing performed must be underpinned by evidence that serious conditions can be prevented, and that the chance of a singleton pregnancy with fewer complications culminating in the delivery of a healthy term infant is increased. The link between the infertility specialist, the general clinician and the couple must be complemented by a comprehensive report from the laboratory embryologists in which detection rates and real-world clinical outcomes of the methods utilized are 
clearly documented, so that the clinicians and couples are able to make informed choices about the benefits and risks of pre-implantation genetic testing specific to their circumstances.

Furthermore, questions remain about the optimal prenatal care of couples whose pregnancies have been achieved following pre-implantation genetic screening. What is the optimal prenatal aneuploidy screening test for pregnancies conceived with PGS? How do first trimester combined aneuploidy screening and cell-free fetal DNA (cffDNA) testing perform in this population, who would presumably have a very low a priori risk of aneuploidy? Should cffDNA testing be offered if the conventional prenatal screening test returns a high riskresult following PGS: how many screening tests are appropriate? If a structural anomaly is identified in a pregnancy conceived with PGS, what is the additional utility of molecular karyotyping on amniotic fluid if, for example, a SNP array was performed as part of PGS? Answers to these questions are not yet forthcoming in the literature, highlighting the need for further research in this field and for detailed reporting of the outcomes of artificial reproductive care. In the meantime, patients deserve detailed and honest appraisals of the limitations of all of these testing regimens and technologies, to ensure they retain informed control over their reproductive choices.

\section{Acknowledgements}

Sammya Bezerra Maia and Holanda Moura are PhD scholar of CAPES (Coordenação de Aperfeiçoamento de Pessoal de Nível Superior, Brazil, scholarship 013982/2013-03).

\section{References}

1. Balasch J, Gratacós E. Delayed childbearing: effects on fertility and the outcome of pregnancy. Curr Opin Obstet Gynecol. 2012;24(3):187-93.

2. Meldrum DR. Introduction: preimplantation genetic screening is alive and very well. Fertil Steril. 2013;100(3):593-4.

3. Mastenbroek S, Twisk M, van der Veen F, Repping S. Preimplantation genetic screening: a systematic review and meta-analysis of RCTs. Hum Reprod Update. $2011 ; 17(4): 454-66$.

4. Simpson JL. Preimplantation genetic diagnosis to improve pregnancy outcomes in subfertility. Best Pract Res Clin Obstet Gynaecol. 2012;26(6):805-15.

5. Iwarsson E, Malmgren, Blennow E. Preimplantation genetic diagnosis: twenty years of practice. Semin Fetal Neonatal Med. $2011 ; 16(2): 74-80$.

6. Anderson RA, Pickering S. The current status of preimplantation genetic screening: British Fertility Society policy and practice guidelines. Hum Fertil (Camb). 2008; 11 (2):71-5.

7. Practice Committee of Society for Assisted Reproductive Technology; Practice Committee of American Society of Reproductive Medicine. Preimplantation genetic testing: a Practice Committee opinion. Fertil Steril. 2008;90(5 Suppl):S136-43.

8. Fischer J, Colls P, Escudero T, Munné S. Preimplantation genetic diagnosis (PGD) improves pregnancy outcome for translocation carriers with a history of recurrent losses. Fertil Steril. 2010;94(1):283-9.

9. Otani T, Roche M, Mizuike M, Colls P, Escudero T, Munné S. Preimplantation genetic diagnosis significantly improves the pregnancy outcome of translocation carriers with a history of recurrent miscarriage and unsuccessful pregnancies. Reprod Biomed Online.2006;13(6):869-74.

10. Samuel GN, Strong KA, Kerridge I, Jordens CF, Ankeny RA, Shaw PJ. Establishing the role of pre-implantation genetic diagnosis with human leucocyte antigen typing: what place do "saviour siblings" have in paediatric transplantation? Arch Dis Child. 2009;94(4):317-20.

11. de Wert G, Liebaers I, Van de Velde H. The future revolution of preimplantation genetic diagnosis/human leukocyte antigen testing: ethical reflections. Stem Cells. 2007;25(9):2167-72.

12. Handyside AH. 24-chromosome copy number analysis: a comparison of available technologies. Fertil Steril. 2013;100(3):595-602.

13. Scott RT Jr, Upham KM, Forman EJ, Zhao T, Treff NR. Cleavage-stage biopsy significantly impairs human embryonic implantation potential while blastocyst biopsy does not: a randomized and paired clinical trial. Fertil Steril. 2013;100(3):624-30.

14. Scott RT Jr, Upham KM, Forman EJ, Hong KH, Scott KL, Taylor D, et al. Blastocyst biopsy with comprehensive chromosome screening and fresh embryo transfer significantly increases in vitro fertilization implantation and delivery rates: a randomized controlled trial. Fertil Steril. 2013;100(3):697-703.

15. Scott KL, Hong KH, Scott RT Jr. Selecting the optimal time to perform biopsy for preimplantation genetic testing. Fertil Steril. 2013; 100(3):608-14.

16. Scott RT Jr, Ferry K, Su J, Tao X, Scott K, Treff NR. Comprehensive chromosome screening is highly predictive of the reproductive potential of human embryos: a prospective, blinded, nonselection study. Fertil Steril. 2012;97(4):870-5.

17. Forman EJ, Treff NR, Stevens JM, Garnsey HM, Katz-Jaffe MG, Scott RT Jr, et al. Embryos whose polar bodies contain reciprocal chromosome aneuploidy are almost always euploid. Hum Reprod. 2013;28(2):502-8.

18. Northrop LE, Treff NR, Levy B, Scott RT Jr. SNP microarray-based 24 chromosome aneuploidy screening demonstrates that cleavage-stage FISH poorly predicts aneuploidy in embryos that develop to morphologically normal blastocysts. Mol Hum Reprod. 2010;16(8):590-600. 
19. Haddad G, He W, Gill J, Witz C, Wang C, Kaskar K, et al. Mosaic pregnancy after transfer of a "euploid" blastocyst screened by DNA microarray. J Ovarian Res. 2013;6(1):70.

20. Bormann CL, Racowsky C. Is universal application of blastocyst biopsy with comprehensive chromosome screening for embryo selection ready for prime time? Fertil Steril. 2013;100(2):e5-6.

21. Menezes M, Meagher S, Costa FS. Ethical considerations when offering non-invasive prenatal testing. Rev Bras Ginecol Obstet. 2013;35(5):195-8.

22. Tao X, Su J, Pepe R, Northrop LE, Ferry KM, Treff NR. PGD for monogenic disease by direct mutation analysis alone in 2 or more cells is more reliable than multiple marker analysis in single cells. Fertil Steril. 2011;96(3 Suppl):S21.

23. Liebaers I, Desmyttere S, Verpoest W, De Rycke M, Staessen C, Sermon K, et al. Report on a consecutive series of 581 children born after blastomere biopsy for preimplantation genetic diagnosis. Hum Reprod. 2010;25(1):275-82.

24. Harper JC, Sengupta SB. Preimplantation genetic diagnosis: state of the art 2011. Hum Genet. 2012;131(2):175-86.

25. Goldsammler M, Jotkowitz A. The ethics of PGD: what about the physician? Am J Bioeth. 2012;12(4):28-9.

26. Majumder MA. More mud, less crystal? Ambivalence, disability, and PGD. Am J Bioeth. 2012;12(4):26-8. 